\title{
Technè
}

La science au service de l'histoire de l'art et de la préservation des biens culturels

40 | 2014

Thérapéia. Polychromie et restauration de la sculpture dans l'Antiquité

\section{Réparations, réfections, modifications de statues- portraits grecques : un volet du projet ANR-DFG EIKON « La vie des portraits grecs »}

Repairs, restoration, modifications made to Greek portrait statues: part of the ANR-DFG project "EIKON: The Life of Greek Portraits"

\section{Martin Szewczyk}

\section{(2) OpenEdition}

\section{Journals}

Édition électronique

URL : https://journals.openedition.org/techne/4033

DOI : $10.4000 /$ techne.4033

ISSN : 2534-5168

\section{Éditeur}

C2RMF

\section{Édition imprimée}

Date de publication : 26 novembre 2014

Pagination : 132-134

ISBN : 978-2-7118-6218-4

ISSN : $1254-7867$

Référence électronique

Martin Szewczyk, «Réparations, réfections, modifications de statues-portraits grecques : un volet du projet ANR-DFG EIKON « La vie des portraits grecs » », Technè [En ligne], 40 | 2014, mis en ligne le 24 juillet 2020, consulté le 08 février 2022. URL : http://journals.openedition.org/techne/4033 ; DOI : https://doi.org/10.4000/techne.4033

\section{(c)}

La revue Technè. La science au service de l'histoire de l'art et de la préservation des biens culturels est mise à disposition selon les termes de la Licence Creative Commons Attribution - Pas d'Utilisation Commerciale - Pas de Modification 4.0 International. 
Martin Szewczyk

\section{Réparations, réfections, modifications de statues-portraits grecques : un volet du projet ANR-DFG EIKON «La vie des portraits grecs »}

Repairs, restoration, modifications made to Greek portrait statues: part of the ANR-DFG project "EIKON: The Life of Greek Portraits"
Résumé. Dans le cadre du projet ANR-DFG EIKON, une étude porte précisément sur les réparations, modifications et transformations de statues-portraits grecques. Cette recherche sintéresse à la fois aux témoignages épigraphiques de telles interventions et aux attestations archéologiques et techniques qui nous en sont parvenues. Plusieurs cas de figure sont présentés ici afin de donner une idée de cette recherche en cours.

Mots-clés. Statue, portrait, réparation, restauration, Grèce antique.

Le projet ANR-DFG EIKON, porté par l'École Pratique des Hautes Études et par l'université de Fribourg-en-Brisgau ${ }^{1}$, a pour ambition de mettre l'accent sur un champ encore peu étudié du portrait grec sculpté: non leur production ou leur exposition première, mais leur «vie », c'est-à-dire l'ensemble des actions qui peuvent affecter le portrait ou le sens dont il est investi dans la société. Cela regroupe donc les performances, cultuelles (rituels...) ou civiques (couronnements, distributions à côté de la statue du bienfaiteur...), les réparations, réfections et transformations, ainsi que les recontextualisations ${ }^{2}$. Le projet doit se conclure par l'édition d'un ouvrage collectif de synthèse, et un colloque, dont les actes seront publiés, sera organisé sur le sujet ${ }^{3}$.

Monuments le plus souvent publics, les portraits grecs ont subi, durant leur existence, ce que l'on appellerait, en langage moderne de la restauration, des «altérations », dues à l'action de la nature ou à celle des hommes, leur causant dommages et modifications dans leur substance et leur apparence; ils ont connu aussi des réparations, en vue de corriger les
Abstract. Within the framework of the ANR/DFG project "EIKON", a study concentrated on the repairs, modifications and changes made to Greek portrait statues. This research examined both the epigraphic evidence of such operations and the archaeological and technical testimony that has come down to us. Several cases are presented here to provide an idea of the research in progress.

Keywords. Statue, portrait, repair, restoration, Ancient Greece.

dégradations ou de redonner du lustre à une statue vieillie, de relever les images abattues par les hommes, voire de modifier de façon intentionnelle le sens donné à l'objet.

Les sources épigraphiques nous font connaître un grand nombre de réparations ou de réfections sur les statues-portraits. Le cas le plus remarquable est celui du marché de réparations commandé à Délos à Aristandros de Paros, sur trois monuments représentant des magistrats romains, sculptés par Agasias d'Éphèse au tournant des $\mathrm{II}^{\mathrm{e}}$ et I ${ }^{\mathrm{er}}$ siècles av. J.-C. ${ }^{4}$ dans l'Agora des Italiens ${ }^{5}$. La première est la statue de Munatius Plancus, dans une niche située sur le côté nord de l'édifice ${ }^{6}$; la seconde, celle de Cluvius, à l'opposé ${ }^{7}$; la troisième, hors contexte, est documentée par la base de statue de Caius Billienus, conservée à Leyde ${ }^{8}$. Dans tous les cas, le Parien a placé sa signature sous celle d'Agasias, substituant

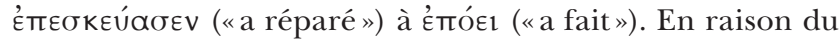
caractère très ciblé des réparations en termes de localisation (exclusivement l'Agora des Italiens) et de personnages représentés (des magistrats romains), il est tentant de situer cette campagne de réparations juste après l'occupation de l'île par

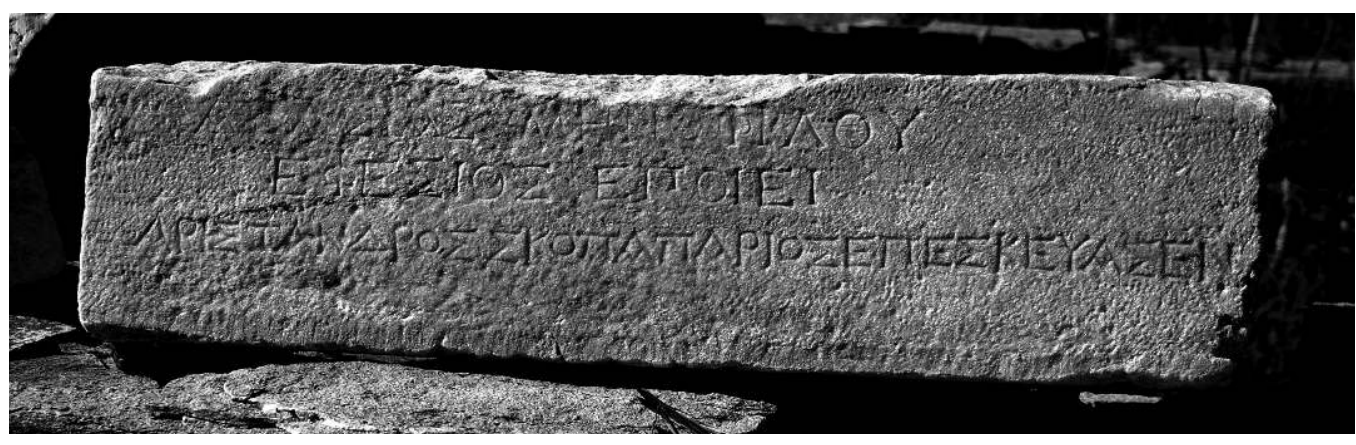

Fig. 1. Bloc de marbre portant les signatures d'Agasias d'Éphèse et d'Aristandros de Paros ; Délos, Agora des Italiens, niche de Cluvius. (C) Martin Szewczyk. 
les troupes pontiques commandées par Archélaos, de mars 87 av. J.-C. à l'automne de la même année ${ }^{9}$. Mais ce dossier exceptionnel ne nous livre le nom que du praticien chargé de la réparation. À l'extrémité orientale du portique d'Antigone, le monument de Caius Billienus a été restauré grâce à la libéralité d'Aulus Attiolenus, citoyen romain d'origine italienne. En temps normaux, dans le sanctuaire, c'eût été à l'épimélète (magistrat athénien, administrateur de l'île, qui avait autorité sur la gestion du sanctuaire et en particulier sur celle des offrandes) de prendre en charge cette intervention ${ }^{10}$. Nous ne savons quelle a été l'ampleur des travaux (l'architecture de la base date bien de la période d'érection du monument, vers 100 av. J.-C.), mais la statue présente des traces de réparation sur le bras gauche (un crampon en fer sur la face extérieure du bras), qui se distingue nettement des techniques d'assemblages par scellement attestées sur l'île à la même époque ${ }^{11}$. Hors contexte cette fois, la statue de Lucius Caecilius Metellus, consul en 113 av. J.-C. ${ }^{12}$, a été restaurée par l'épimélète - un Athénien, donc - Protimos, fils de Dosithéos. Cela date l'intervention de 80 environ, après le rétablissement du pouvoir athénien sur l'île, et nous indique que la cité, et pas seulement les Italiens présents sur l'île, étaient concernés par la restauration des monuments attaqués lors de la guerre mithridatique.

Les décrets concernant des restaurations peuvent nous en apprendre beaucoup sur les agents et leurs motivations. Ainsi, le décret d'Érythrées, que l'on peut situer au début du III $^{\mathrm{e}}$ siècle av. J.-C., concernant la réfection de la statue, en bronze, du tyrannicide Philitas ${ }^{13}$, signale que le portrait du grand homme s'est vu amputé de son épée par les partisans de l'oligarchie, qui estimaient que l'érection de la statue les visait directement. Le Conseil et le Peuple décident alors que les exetastai (un collège de contrôleurs financiers chargés, en particulier, de l'adjudication des travaux publics) veilleront à redonner à la statue son intégrité:

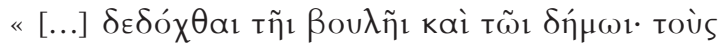

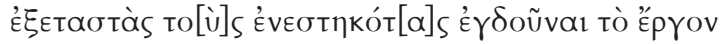

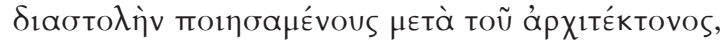

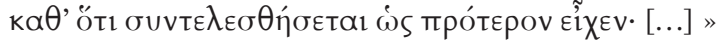

«Il a été décidé par le Conseil et le Peuple: les exetastai en charge doivent passer commande du travail auprès de l'architecte, de façon à contribuer ensemble à compléter la statue comme dans son état initial. » (1. 10-14).

Les agoranomes en entretiendront par ailleurs le bon état de surface (en éliminant l'oxydation du cuivre (en grec ios) ; 1. 14-17). L'enjeu est celui du respect et de l'entretien de la mémoire des bienfaiteurs de la patrie (1. 6-9).

Parce que les monuments inscrits sont autant des objets que des textes, nous pourrons nous pencher sur les indices techniques ou archéologiques qu'ils nous présentent, portant dans leur matérialité même les traces d'interventions diverses. Ainsi, à Pergame, un bloc correspondant, dans son dernier état, à un orthostate, découvert en quatre fragments dans le sanctuaire d'Athéna et sur l'Agora entre 1879 et $1883^{14}$, présente trois états d'usage. Le dernier est documenté par l'inscription commémorant la réparation et le rétablissement, par «le Conseil et le Peuple ${ }^{15}$ », du monument dédié par les marins de la flotte militaire d'Eumène après la guerre contre Nabis et Antiochos, probablement en 191 av. J.-C. ${ }^{16}$ Le bloc présente, sur sa face postérieure, trois semelles de forme circulaire, correspondant probablement à une statue équestre, et sur la face latérale, le reste d'une première inscription. Cela témoigne à la fois de la réutilisation des dispositifs architecturaux des statues et de leur nécessaire restauration par les pouvoirs publics.

Les exemples concrets, touchant à la matérialité des statues elles-mêmes, sont bien plus sporadiques. Ces témoignages sont néanmoins précieux afin d'étudier la mise en œuvre des interventions dont les motivations et les applications pratiques (les procédures, en particulier) ont pu être circonscrites de manière générale par les témoignages épigraphiques. Le portrait de roi attalide découvert dans le gymnase de Pergame, et identifié comme Eumène II (ou Attale I ${ }^{\mathrm{er}}$ ), témoigne d'une réfection: les cheveux au-dessus du front et des tempes ont été resculptés et les surfaces apprêtées afin de placer les ajouts en marbre, d'une facture différente (travail de délimitation des touffes au foret et usage du ciseau pour les détails) du reste de la chevelure. Cette réfection, conférant à l'image royale un aspect alexandrin, a été diversement datée, du règne d'Attale $I^{\text {er }}$ jusqu'au I ${ }^{\text {er }}$ siècle av. J.-C. En revanche, d'après certaines restitutions d'un décret de Pergame en l'honneur

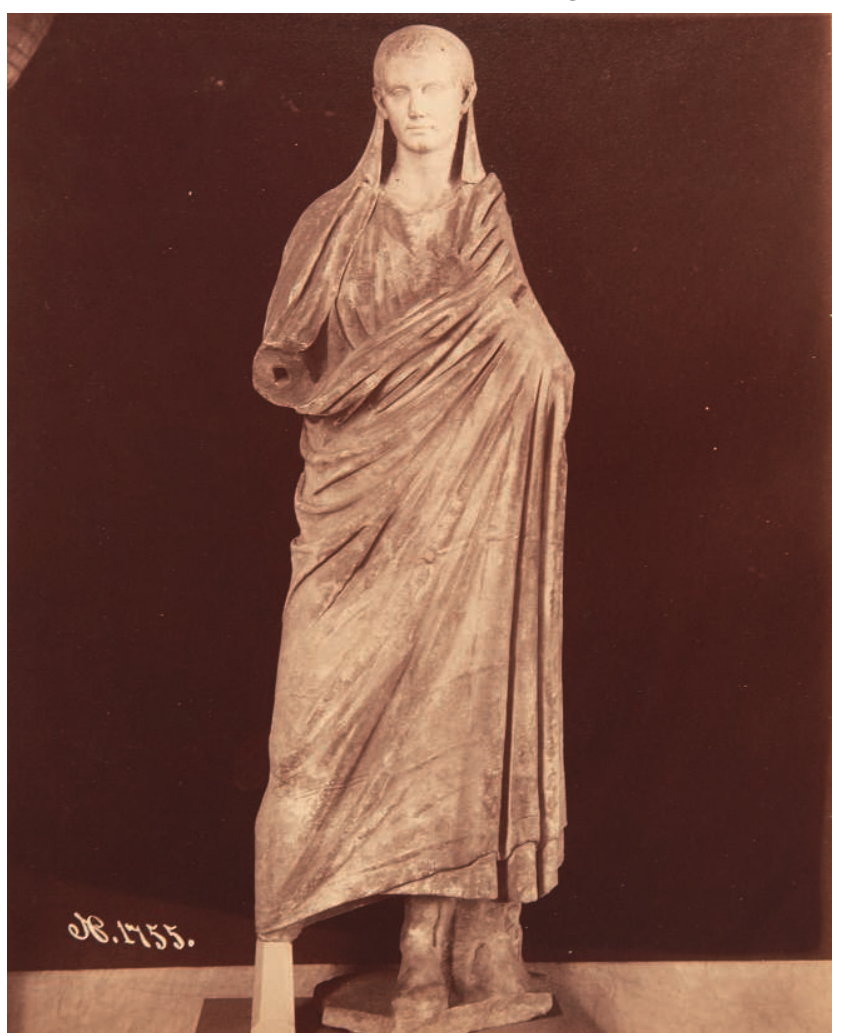

Fig. 2. Statue masculine drapée, Istanbul, Musée archéologique, inv. 710 ; découverte à Magnésie du Sipyle en février 1895, marbre, H. : 2,13 m (plinthe : 0,70 m), vers 150 av. J.-C. (C) Fond Mendel/Sebah \& Joaillier, Musées archéologiques d'Istanbul (numérisation G. Paquot, EPHE-INHA). 
du bienfaiteur Diodoros Pasparos, celui-ci aurait réparé, vers 69 av. J.-C., les agalmata des rois dans le gymnase ${ }^{17}$. Certains fragments de statues colossales, notamment des pieds, témoignent également de réfections ${ }^{18}$. La pratique de la métarythmèsis (changement de la tête d'une statue-portrait afin de modifier son identification ${ }^{19}$ ), documentée par les sources littéraires, nous est également connue grâce à des vestiges statuaires: ainsi une statue masculine découverte à Magnésie du Sipyle, aujourd'hui à Istanbul ${ }^{20}$. L'examen de la pièce révèle que la tête capite velato, stylistiquement datée de l'époque augustéenne, a été ajustée dans un second temps sur une statue d'époque hellénistique portant l'himation; la discordance entre un manteau grec et un attribut de l'officiant du ritus romanus révèle le réemploi d'une statue-portrait anté-

134 rieure, peut-être d'un notable grec, certainement pour honorer un magistrat romain.

En poursuivant les investigations et en tâchant de dégager au mieux les motivations et les pratiques associées à la réparation et à la réfection de statues-portraits, on pourra situer la question sur la toile de fond d'un débat moderne: la distinction qui s'est opérée petit à petit, sur le plan des pratiques, celui des idées et celui des institutions, entre réparation d'un côté et restauration de l'autre. Cette histoire moderne peut nous permettre d'éclairer des attitudes vis-à-vis de ce que nous considérons comme «œuvres d'art», mais qui étaient

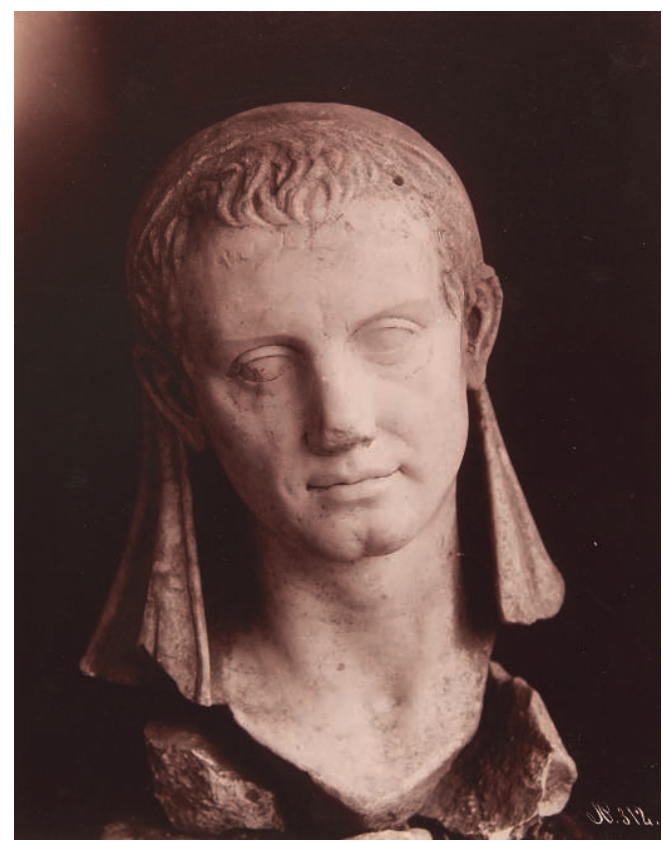

Fig. 3. Tête rapportée de la statue masculine drapée de Magnésie du Sipyle, années 20-10 av. J.-C. (C) Fond Mendel/Sebah \& Joaillier, Musées archéologiques d'Istanbul (numérisation G. Paquot, EPHE-INHA). alors perçues à l'aune de leur rôle social et public.

\section{Notes}

Liste des sigles et abréviations de collections ID: Corpus des Inscriptions de Délos, Paris. $I G$ : Inscriptiones Graecae, Berlin.

IGR: Inscriptiones Graecae ad res Romanas pertinentes.

1. Piloté par François Queyrel (EPHE) et Ralf von den Hoff (Universität Freiburg).

2. Dans ce texte, j'entends par

«réparation» toute intervention visant à rétablir l'état originel d'une œuvre ou d'un monument (du latin reparare, «réparer, remettre sur pied») et portant sur la matière originelle; par «réfection ", celle de modifier intentionnellement une œuvre, par retaille, réintégration d'un élément perdu ou d'un attribut nouveau. Du fait de la spécialisation moderne du terme «restauration », je l'emploie plutôt ici dans un sens général, plus proche du vocabulaire antique (les verbes et substantifs pouvant être utilisés pour un monument comme pour un rituel ou un régime politique), indiquant l'intention de redonner à l'objet son rôle social et public. Il faut bien avoir à l'esprit que la terminologie latine, dont est issu le vocabulaire français, ne présente pas une différentiation sémantique très nette; par ailleurs, on ne peut guère dire dans quelle mesure il présente une congruence avec le vocabulaire grec.

3. Pour plus d'informations: http://www.eikon-anrdfg.eu/.

4. Sur la carrière d'Agasias d'Éphèse: Picard, 1910

5. Sur ce monument: Lapalus, 1939; Trümper, 2008.
6. ID 1697. Il est à remarquer que la signature d'Aristandros est gravée sur un des deux blocs formant la banquette de l'exèdre, blocs visiblement réemployés (moulure rabattue).

7. ID 2494.

8. ID 1717.

9. Bruneau, 1968, p. 671-673; sur la chronologie: Baslez, 1983.

10. Nous nous situons donc certainement lors de la période de perte de contrôle de l'île par Athènes, entre l'automne 87 et la fin des années 80 .

11. À ce sujet, voir la thèse de Ph. Jockey, Techniques et ateliers de sculpture à Délos à l'époque hellénistique (Thèse, Université Paris X, 1993), en particulier p. 240-249.

12. Homolle, 1879 , p. $158, \mathrm{n}^{\circ} 5$.

13. I. Erythrai 503; Heisserer, 1979.

14. IvP I $62 ; \mathrm{L}: 1,705 ; \mathrm{H}: 0,940$; Pr: 0,220; quatre fragments; le lit d'attente présente deux trous de crampons; la face postérieure présente des trous d'attache pour une statue équestre en bronze.

15. Ce qui date certainement la chose après le règne de Tibère.

16. Will, 1982, p. 211-212.

17. IGR IV 294, 1. 19-20. Au vu de la date tardive et de la nature des interventions de Diodoros Pasparos dans le gymnase, on privilégiera une réparation: Queyrel, 2003, p. 37.

18. Je dois cette information à R. von den Hoff.

19. À ce sujet, voir Blanck 1969.

20. Mendel, 1914, n 591 ; Inan, Rosenbaum, 1966, n 209, pl. 115,1 et 116 , 1-2.

\section{Bibliographie}

Baslez M.-F., 1983, «Délos pendant la première guerre de Mithridate », dans Solin H., Coarelli F., Musti D., Delo e l'Italia, Rome, p. 51-66.

Blanck H., 1969, Wiederverwendung alter Statuenals Ehrendenkmäler bei Griechen und Römern, Rome.

Bruneau Ph., 1968, "Contribution à l'histoire urbaine de Délos », BCH, 92, p. 633-709.

Heisserer A. J., 1979, “The Philites Stele $\left(\mathrm{SIG}^{3} 284\right.$ = IEK 503)", Hesperia, 48-3, p. $281-293$

Homolle Th., 1879, «Inscriptions de Délos », $B C H, 3$, p. $158, \mathrm{n}^{\circ} 5$.

Inan J., Rosenbaum E., 1966, Roman and Early Byzantine Portrait Sculpture in Asia Minor, Londres.

Lapalus E., 1939, L'Agora des Italiens (EAD 19), éd. de Boccard, Paris.

Mendel G., 1914, Catalogue des sculptures grecques, romaines et byzantines, II, Constantinople.

Picard Ch., 1910, «Le sculpteur Agasias d'Éphèse à Délos ", $B C H, 34$, p. 538548.

Queyrel F., 2003, Les portraits des Attalides, Fonction et représentation, éd. de Boccard, Paris.

Trümper M., 2008, Die 'Agora des Italiens' in Delos: Baugeschichte, Architektur, Ausstattung und Funktion einer späthellenistischen Porticus-Anlage, Rahden.

Will E., 1982, Histoire politique du monde hellénistique, 323-30 av. J.-C., II, Seuil, Paris. 